
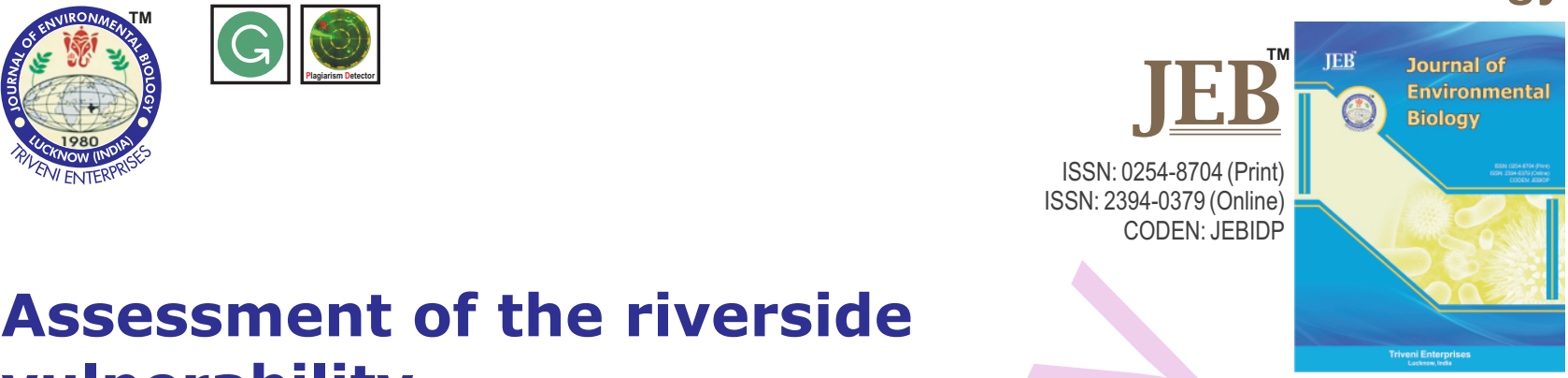

\title{
Assessment of the riverside vulnerability
}

\section{Authors Info}

D. Kereselidze ${ }^{1}$, L. Matchavariani ${ }^{2 *}$, V. Trapaidze ${ }^{1}$ and G. Bregvadze ${ }^{1}$

'Department of Hydrometeorology, Faculty of Exact and Natural Sciences, Tbilisi State University, 0179, Tbilisi, Georgia

${ }^{2}$ Department of Soil Geography, Faculty of Exact and Natural Sciences, Tbilisi State University, 0179, Tbilisi, Georgia

*Corresponding Author Email : lia.matchavariani@tsu.ge

\section{Key words}

Random processes,

Reliability theory,

Risk forecasting,

Riverbed degradation,

Riverside vulnerability

\section{Publication Info}

Paper received : 30.07 .2016

Revised received : 25.06.2017

Accepted : 28.06.2017

\section{Abstract}

Aim: Principal objective of the present work was to consider the mechanism of riverside destruction by water flow as a random process, which depends on the flow velocity and sustainability of the riverside. The riverside vulnerability was taken as the indicator with respect to the influence of such processes.

Methodology: Vulnerability assessed according to all those indices characteristic of its critical value. It was necessary to select one of the basic determining (integral) factors out of the indicators characteristic of critical state, according to which vulnerability of the object will be assessed.

Results: With the aim of practical application of the considered method, let us examine a virtual example was examined. The observation data subject to normal distribution law was assumed. For the admissible river current velocity $n_{a}=15$, mathematical expectation $m_{a}=3 \mathrm{~m} \mathrm{sec}^{-1}$, mean square deviation $\sigma_{a}=0.9$; for bed velocity, $n_{b}=20$. The confidence interval of bank vulnerability was identified. Finally, the vulnerability of a river bank with 0.95 probability was within the interval of $\mathrm{I}_{0.95}=(0.57 ; 0.9)$ with point evaluation $z=0.77$.

Interpretation: The mechanism of riverside destruction by water flow was considered as a random process, which depends on both the effect of flow rate and riverside stability. Vulnerability, the characteristic of the riverside was taken as the indicator of this process with respect to such influence, and famous model "load - stability" from the theory of reliability was used for its identification.

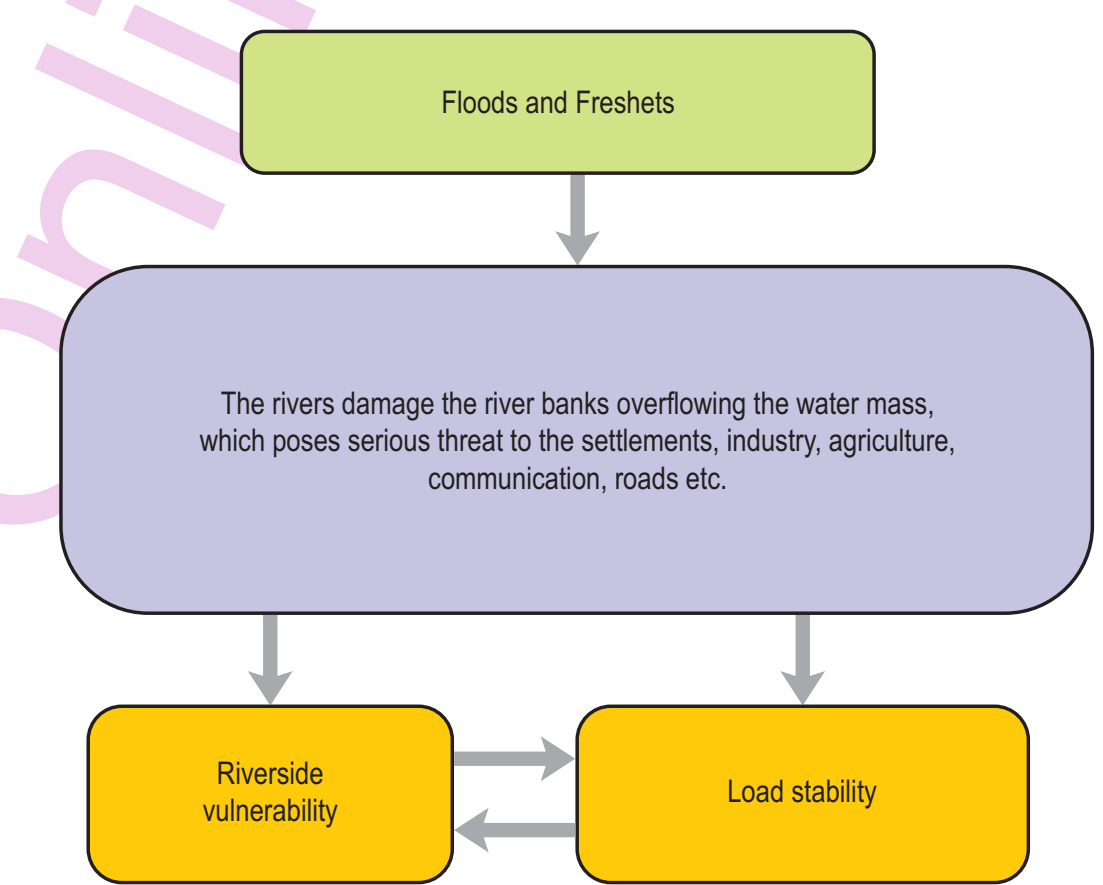




\section{Introduction}

The world is facing unprecedented global changes. Areas that are vital for existence of human beings suffer great changes threatening the well-being of the present and future generations. According to UN experts, recent natural disasters, such as floods and freshets, occurring against the background of climate change are followed by certain ecological problems and socio-economic difficulties.

Since ancient time, people have combatted the freshets and their negative consequences in different ways and methods. From the point of chronology, at first it was just based on the descriptive and empirical studies lacking scientific foundations of problem solution but at present hydrology is the science that can make qualitative assessment and forecast an event.

Nowadays, in many countries worldwide the researches on the riverside vulnerability (Oyer Zachary, 2014), sustainability of water ecosystems (Simeoni and Corinne, 2009; DePhilip Michele and Moberg, 2010) as well as flood control (Pielke, 2000; Shannon, 2010; Rashid, 2011), evaluation of freshet risks (SaintLaurent Diane, 2007; Lebel and Bach, 2009; Burrell et al., 2015), water quality (Ronghui Li et al., 2014), changes and use of river delta (Hüseyin Korkmaz et al., 2012), etc. are intensive.

Although, we have number of fundamental scientific studies on forecasting the risks of floods and freshets, the problem cannot be condsidered to be completely solved. Recently, in hydrological forecasting a certain progress is observed in application of modern mechanism of the theory of random processes and fields.

Study area : The problem of floods and freshets is topical in Georgia. Against the diverse orographic background of its territory, over 26000 small and medium rivers are registered with the majority flowing in Western Georgia. Very often the rivers damage the river banks overflowing the water mass, which poses serious threat to the settlements, industry, agriculture, communication, roads, etc.

In most cases the water overflows from the river due to the riverside damage and its malfunctioning as water-retainer. A clear example is the well-known freshet on the river Rioni in 1987, when the intensive lateral erosion took place due to the strong water flow, damaged the dams and, in consequence, a two-meter high wave flooded the settlements, arable lands, communication, road network and destroyed lots of houses and even took human lives. Above-given fact and many others require preliminary assessment of riverside vulnerability. Therefore, principal objective of the present work is to consider the mechanism of riverside destruction by water flow as a random process, which depends on the flow velocity and sustainability of the riverside. The riverside vulnerability is taken as the indicator with respect to the influence of such processes.

\section{Materials and Methods}

Identification of point wise values of vulnerability: The vulnerability degree depends on the load intensity and its duration and the relationship between the components of the object. The critical limit of vulnerability conditions the range of trouble-free operation of the object. Vulnerability should be assessed according to all those indices characteristic of its critical value that is not feasible due to the limited processes of exact analytical modelling. Therefore, it is necessary to select one of the basic determining (integral) factors out of the indicators characteristic of critical state, according to which vulnerability of the object will be assessed.

After selection of the determining factor, it is possible to use the well-known procedures of reliability assessment for estimation of vulnerability. To this end, in the theory of reliability the model of "load-strength" is often used (Mirtskhulava, 2003; Bazovsky, 2004; Høyland Arnljot and Marvin Rausand, 2009; Jardine and Tsang, 2013; Gnedenko et al., 2014).

In our case the bottom velocity of the water flow $(\mathrm{Vb})$ can be considered as a determining factor in regard to "load". Approximate values of the bottom velocity for any cross-section can be defined by consideration of the values of morphometrical characteristics, hydrological and hydraulic elements of the river bed, which can be defined more precisely by means of direct measurements if necessary, while with respect to the riverside, the determining factor will be the integral feature of the ground counter action determining the permissible velocity of water flow. The latter is the maximum value of water flow, which does not cause the ground washing-out.

Let us consider a short, straight-line stretch of the river bed, which can be considered as prismatic with uniform riverside grounds. Under this assumption we might formalize the problem of riverside vulnerability assessment to some extent. Let us assume that in a certain time period (decade) the river movement on the given site is steady and the bottom velocity $\left(V_{b}\right)$ experiences relatively insignificant changes. Under these conditions the bottom velociy of the flow can be considered as a random value $V_{b}$, the distribution density of which is $f_{1}\left(V_{b}\right)$, mathematical expectation is $m_{v b}$ and mean square deviation is $\delta_{v b}$.

In the same way, the permissible speed of water flow $\mathrm{V}_{\mathrm{p}}$ can be considered as a random value, the distribution density of which is $f_{2}\left(V_{p}\right)$, mathematical expectation is $m_{v p}$ and the mean square deviation is $\delta_{v p}$. Naturally, if $V_{b}$ exceeds $V_{p}$, the riverside washing-off will take place, i.e. mutual consideration of random values $V_{b}$ and $V_{p}$ as a system allows to forecast the riverside vulnerability. To this end, let us introduce a new random value $Z$, which is related to our initial random values with a general functional relation: 


$$
Z=f\left(V_{b}, V_{p}\right)
$$

Let the probability density $f\left(V_{b}-V_{p}\right)$ be a known system of random values $\left(V_{b}, V_{p}\right)$ and determine the distribution law for $Z$. With that, the problem of the vulnerability forecasting is considerably simplified, since from the system of two random values we have one random value $Z$.

As far as the relation (1) represents a certain surface in $\mathrm{V}_{b} 0 \mathrm{~V}_{\mathrm{p}} \mathrm{Z}$ system, the distribution function $\mathrm{G}(\mathrm{z})$ of random value $\mathrm{Z}$ (Fig.1A) will be written as follows (Mirtskhulava, 2003; Rausand and Høyland, 2004; Thoft-Cristensen and Baker, 2012; Gertsbakh, 2013; Gnedenko et al., 2014):

$$
G(z)=P(Z<z)=P\left(f\left(V_{b}, V_{p}\right)<z\right)
$$

where $\mathrm{z}$ is a distance from the crossing plane $\mathrm{H}$ to coordinate plane $\mathrm{V}_{b} O \mathrm{~V}_{\mathrm{p}}$, which is drawn in parallel to its secant line $\mathrm{K}$, the points of which satisfy the equality $\varphi\left(V_{b}, V_{p}\right)=Z$, the latter will be devided into two areas by $\mathrm{V}_{b} \mathrm{OV}_{\mathrm{p}}$ while projecting on to the coordinate plane. The points of one of them (let us denote it by $\mathrm{D}$ ) satisfy the condition $\varphi\left(V_{b}, V_{p}\right)<Z$. Then, for satisfying (2) the a random point $\left(V_{b}, V_{p}\right)$ must get into $D$ area; proceeding from this fact we can write:

$$
G(z)=P\left(\left(V_{b}, V_{p}\right)<D\right)=\iint_{(D)} f\left(V_{b}, V_{p}\right) d v_{b} d v_{p}
$$

In this mathematical expression the parameter $z$ is implicitly within the integration limits. After differentiation of $G(z)$ on $z$ we will get the distribution density for random value $Z$. $g(z)=G^{\prime}(z)$

Since the concrete form of function $Z=f\left(V_{b}, V_{p}\right)$ is known, integration limits can be given by $z$ and the expression $g(z)$ can be written in an explicit form. As far as the difference between $V_{p}$ and $V_{b}$ is significant for forecasting the riverside vulnerability, it is necessary to consider functional relation $Z=\varphi\left(V_{p}, V_{b}\right)$ in the form of difference between random values $Z=\left(V_{p}-V_{b}\right)$.
In this case $D$ area will be represented as a semi-plane of the coordinate plane $\left(\mathrm{V}_{b} 0 \mathrm{~V}_{\mathrm{p}}\right)$, which lies below the straight line $\left(\mathrm{V}_{\mathrm{p}}\right.$ $\left.V_{b}\right)=Z$. This line cuts coordinate axes into $Z$ congruent segments (in positive and negative directions) (Fig. 1B).

Random points, which got into this area, satisfy the condition $V_{p}$ $V_{b}<Z$. In this case distribution function (3) will be as follows:

$$
G(z)=\iint_{(D)} f\left(V_{b}, V_{p}\right) d v_{b} d v_{p}=\int+^{\infty}\left\{\int^{Z+v b} f\left(V_{b}, V_{p}\right) d v_{p}\right\}
$$

After differentiation of this expression on $z$ we will get distribution density for random value $Z$

$$
g(z)=\int f\left(V_{b}, z+V_{p}\right) d v_{p}
$$

As far as $V_{b}$ and $V_{p}$ are independent random values, $f\left(V_{b}, V_{p}\right)=$ $\mathrm{f}_{1}\left(\mathrm{~V}_{\mathrm{b}}\right) \mathrm{f}_{2}\left(\mathrm{~V}_{\mathrm{p}}\right)$

Thus, the distribution density (5) for random value $Z$ will be:

$$
g(z)=\int f_{1}\left(V_{p}\right) f_{2}\left(z+V_{p}\right) d v_{p}
$$

In this case it can be said that composition of two laws takes place, which will be written as follows: $g=f_{1}{ }^{*} f_{2}$

In general, distribution laws for $\mathrm{f}_{1}$ and $\mathrm{f}_{2}$ can be of any form, while in our case $V_{b}$ and $V_{p}$ represent the random value of the normal distribution that is confirmed by statistical data of longterm observations.

In this case distribution densities $f_{1}$ and $f_{2}$ will be written in following form:

$$
f_{1}\left(V_{f}\right)=\left(1 / \delta_{v b} \sqrt{ } 2 \pi\right) e^{-((V b-m v b) 2) / 2 \delta} 2 ; \quad f_{2}\left(V_{b}\right)=\left(1 / \delta_{v b} \sqrt{ } 2 \pi\right) e^{-((V d-m v b) 2 / 2 \delta} 2
$$

As is known, composition of two normal distribution laws provides the normal distribution law, at the same time mathematical expectation of new random value is an algebraic sum of initial random values, while dispersion is a sum of
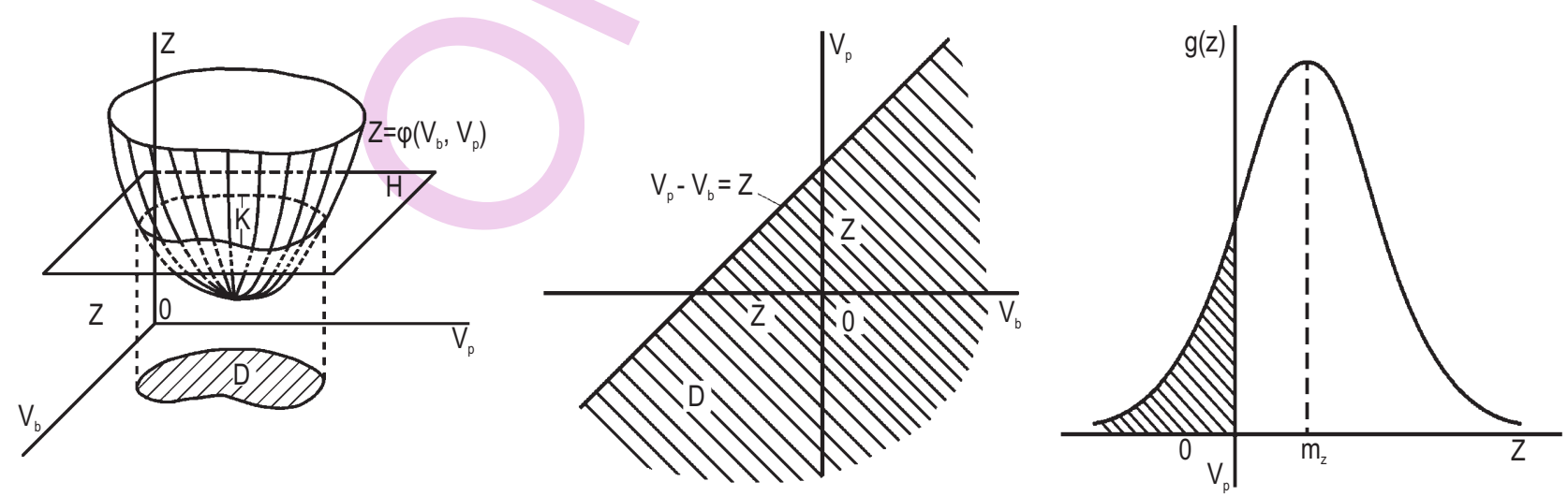

Fig. 1 : Graphic expressions of calculations: $(A)$ function $Z=f(V b, V p)$; $(B)$ disposition of $D$ area on plane and $(C)$ normal distribution function 
dispersions.

In our case we will have: $m z=m v p-m v b, \delta z=\sqrt{\delta} v p+\delta v b$.

Distribution density of random value Z will be as follows:

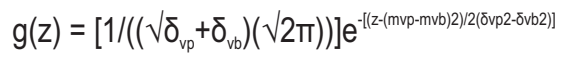

While its distribution function will be: $G(z)=\int^{z} g(z) d z$

As far as there is no risk of riverside washing-off for random value $Z>0$, vulnerability ( $r$ ) will be assessed according to the equation (fig. 1-C):

$$
r=P(Z<0)=G(0)=\int g(z) d z
$$

Analysis of the obtained formulas shows that the increase of $V_{b}$ causes reduction of $m_{z}$, that, in its turn, increases the area under the curve in the negative area of axis $z$ and, therefore, riverside vulnerability assessment at one or another section increases, too.

As far as the distribution function $\mathrm{G}(\mathrm{z})$ with parameters $\left(\mathrm{m}_{\mathrm{z}}, \delta_{\mathrm{z}}\right)$ can be expressed by the normal distribution function $\Phi^{*}$ of random value with parameters $(0.1)$ in the following form:

$$
\mathrm{G}(\mathrm{z})=\Phi^{*}\left(\mathrm{z}-\left(\mathrm{m}_{\mathrm{z}} / \delta_{\mathrm{z}}\right)\right)
$$

Then the value of riverside vulnerability can be computed by the following formula:

$$
r=G(0)=\Phi^{*}\left(-m_{z} / \delta_{z}\right)
$$

Identification of the vulnerability confidence interval : Let us consider the following common problem in advance. Let $X$ be a random quantity with its distribution law containing the unknown parameter $a$. We must find relevant valueof this parameter $a^{\sim}$, which is received from $n$ independent experiments, where $X$ acquires certain values $X_{1}, X_{2}, \ldots X_{n}$. It is clear that given assessment depends on the distribution law of $X$, as well as on the number of experiments ( $\mathrm{n})$, and is therefore, a random quantity with the distribution law of unknown value $\mathrm{a}^{\sim}$. In addition, it is a necessary condition that as $\mathrm{n}$ increases, the assessment approximates parameter a itself by probability, and its mathematical expectation $\mathrm{M}[\mathrm{a}]$ equals $\mathrm{a}$. In particular, for $\mathrm{m}$ the mathematical expectation of $X$ random quantity and $D$ dispersion we will have

$$
m^{\sim}=\sum X / n ; D^{\sim}=\sum\left(X_{i}-m\right)^{2} / n-1
$$

These values for unknown $m$ and $D$ determine the point valuation. Let us note that $m^{\sim}$ coincides with its statistical analog $m^{*}=\sum X_{i} / n$, and $D^{\sim}$ differs from $D^{*}=\sum\left(X_{i}-m^{*}\right) / n-1$. However, as $n$ increases, the difference between $D^{\sim}$ and $D^{*}$ virtually, disappears.

First, let us try to determine the confidence interval for the principal parameters of the random quantity, in particular, for mathematical expectation $\mathrm{m}$, let us take some high probability $\beta\left(0.9,0.95\right.$.. $\beta(0.9,0.95 .$.$) and let us find interval I_{\beta}$, for which the following condition is true:

$$
P\left(\left|m^{\sim}-m\right|<\varepsilon_{\beta}=\beta\right.
$$

where $\varepsilon_{\beta}$ is half of the width of $I_{\beta}$. Values $\beta$ and $I_{\beta}$ are called confidence probability and confidence interval, respectively. If the distribution law of $\mathrm{m}^{\sim}$ had been known, the solution to the problem would have been easy. In case of the unknown distribution law of $\mathrm{m}$, a rough method is used to define the confidence interval, which is based on the assumption that the distribution of random quantity $\mathrm{m}^{\sim}$ is normal notwithstanding the kind of law of distribution of initial random quantity $X$. On the other hand, if admitting that the distribution law of random quantity $X$ is normal, then from random quantity $m^{\sim}$ we can shift to new random quantity T by using the following formula:

$$
T=\sqrt{ } \mathrm{n}\left(\left(\mathrm{m}^{\sim}-\mathrm{m}\right) / \sqrt{ } \mathrm{D}\right)
$$

which obeys to so-called Student's $\mathrm{t}$ - distribution with n-1 degree of freedom. The distribution density of this law is as follows:

$$
S_{n-1}(t)=G\left(\frac{n}{2}\right) / \sqrt{\pi(n-1)} G\left(\frac{n-1}{2}\right) \cdot\left(1+\frac{t^{2}}{n-1}\right)^{-\frac{n}{2}}
$$

where $\mathrm{G}(\mathrm{x})$ is a known as gamma-function:

$$
G(x)=\int u^{x-1} e^{u} d u
$$

By considering (3), expression (2) will be as follows:

$$
P=\left(|T|<\left(\varepsilon_{\beta} / \sqrt{ } D \sim n\right)\right)=\beta \text {. }
$$

By denoting $\varepsilon_{\beta} / \sqrt{ }\left(D^{\sim} / n\right)=t_{\beta}$ we will have: $P\left(|T|<t_{\beta}\right)=\int S_{n-1}(t) d t=\beta$

By considering that $S_{n-1}(t)$ is an even function, finally we will have: $2 \int S_{n-1}(t) d t=\beta$

By solving this equation, the values of $\beta$ given in relation to $t_{\beta}$ and by using the number of $n-1$ degrees of freedom, we will find:

$$
\varepsilon_{\beta}=t_{\beta} \sqrt{ }\left(D^{\sim} / n\right)
$$

In reality, there are tables of Student distribution law, where $t_{\beta}$ is defined according to $\beta$ and $(n-1)$.

The confidence interval for mathematical expectation $\mathrm{m}$ is defined depending on $\varepsilon_{\beta}$, which is symmetrical to $\mathrm{m}^{\sim}$ :

$$
I_{\beta}=\left(m^{\sim}-\varepsilon_{\beta} ; m^{\sim}+\varepsilon_{\beta}\right)
$$

The confidence interval of $X$ random quantity with normal distribution for (D) dispersion is defined similarly. Assessment of (D) dispersion in formula (1) is expressed by means of ( $v$ ) random quantity, which has $\left(X^{2}\right)$ distribution for $(n-1)$ degree of freedom. 


$$
K_{n-1}(\sigma)=\left\{\begin{array}{lll}
\frac{1}{2^{\frac{n-1}{2}} \Gamma\left(\frac{n-1}{2}\right)} \sigma^{\frac{n-1}{2}-1} e^{-\frac{\sigma}{2}}, & \text { when } & \sigma>0 \\
0, & \text { when } & \sigma<0
\end{array}\right\}
$$

As the distribution density of $\mathrm{K}_{n-1}(\sigma)$ is not a symmetrical function, the limits of the confidence interval of $(v)$ random quantity will be selected from the condition of the same probability of the departure from any end of this interval. As the given confidence interval equals $\beta$, the probability of $(v)$ not occurring in the confidence interval, equals $(1-\beta)$. This condition is met, if the probability of $\mathrm{V}$ random value occurring beyond the right and left ends of the interval equals to $a / 2=(1-\beta) / 2$. The tables are drafted for $\left(X^{2}\right)$ distribution, according to which, for $(p)$ probability and $(n-1)$ number of degrees of freedom, the values of $\left(X^{2}\right)$ can be found to meet the following conditions:

$P\left(V>X^{2}\right)=p$.

In our case, for $P_{1}=a / 2$ let us find $\left(x_{1}^{2}\right)$ and for $P_{2}=1-(a / 2)$ let us find $\left(X_{2}^{2}\right)$. Now, we can define $D_{1}$ and $D_{2}$ limits of confidence intervals $D$, which corresponds to $\beta-1$ confidence probability: $P\left(D_{1}<D_{2}<D_{3}\right)=\beta$.

Equation (16) shows that condition $D>D_{1}=D^{\sim}(n-1) / X_{1}^{2} n$ corresponds condition $V<\chi_{1}{ }^{2}$, while condition $D<D_{2}=D^{\sim}(n-1) / X_{2}{ }^{2}$ corresponds to condition $V>X_{2}{ }^{2}$. Thus, the confidence interval for $D$ dispersion of normally distributed $X$ random quantity $I_{\beta}$, which corresponds to $\beta$ confidence probability and $(n-1)$ number of degrees of freedom, will be:

$$
I_{\beta}=\left(\frac{\tilde{D}(n-1)}{\chi_{1}^{2}} ; \frac{\tilde{D}(n-1)}{\chi_{2}^{2}}\right)
$$

Now, we can define the confidence interval for $\mathrm{T}$ value of the river bank, whose point valuation is calculated by the formula:

$$
t=\varphi^{*}\left(-\left(m_{z} / \delta_{z}\right)\right)
$$

Here $Z$ is a random value equalling to the difference between the non-washing (admissible) velocity of the coast $V_{a}$ and $V_{b}$ bed velocity of the water current $\left(z=V_{a}-V_{b}\right)$, while $\varphi^{*}(x)-$ is the normal parameters of the distribution function (0.1). It is implied that $V_{a}$ and $V_{b}$ are normally distributed values with relevant mathematical expectation and mean square deviation $m_{a}, \sigma_{a}$ and $m_{b}, \sigma_{b}$. From here, it is clear that random value $z$ itself subjects to the distribution law with mathematical expectation $m_{z}=m_{a}-m_{b}$ and mean square deviation of:

$$
\delta_{z}=\sqrt{\delta_{\mathrm{a}}^{2}-\delta_{\mathrm{b}}^{2}}
$$

To calculate the values of $\mathrm{T}$ by formula (17), point valuations $\mathrm{m}_{z}$ and $\delta_{z}$ are used for $m_{z}$ mathematical expectation of random quantity $z$ and mean square deviation $\sigma_{z}$, and in line with the formula (11) the following values are defined, $D^{\sim}{ }_{a}$ and, $D^{\sim}$

$$
\begin{aligned}
& \tilde{m}_{a}=\sum_{i=1}^{n_{p}} V_{a i} / n_{a} ; \quad \tilde{D}_{a}=\sum_{i=1}^{n_{p}}\left(V_{a i}-\tilde{m}_{a}\right)^{2} / n_{a}-1 \\
& \tilde{m}_{b}=\sum_{i=1}^{n_{d}} V_{b i} / n_{b} ; \tilde{D}_{b}=\sum_{i=1}^{n_{d}}\left(V_{b i}-\tilde{m}_{b}\right)^{2} / n_{b}-1
\end{aligned}
$$

where $n_{a}$ and $n_{b}$ are the numbers of the observation data of random quantities $V_{a}$ and $V_{b}$, respectively.

By considering $\tilde{m}_{z}$ and $\delta_{z}^{\sim}$ randomness, to strictly define the limits of $Z$ confidence interval, we must consider a new random quantity $V=-m_{z}^{\sim} / \delta_{z}^{\sim}$ and its distribution law must be identified.

After identifying the limits of $m_{y}$ the confidence interval of the mathematical expectation of value, we can fix the limits of the $z$ confidence interval, $z_{1}$ and $z_{2}$, from formula (17): $z_{1}=\varphi^{*}\left(y_{1}\right)$ and $z_{2}$ $=\varphi^{*}\left(\mathrm{y}_{2}\right)$.

Identification of the distribution law of random quantity $y$ entail certain difficulties leading to an unjustified complication of the problem. However, considering the slight dependence of $z$ on the form of distribution density of random quantity z, i.e. $\sigma_{z}$ and essential dependence on the location of the dissipation center, on axis $z$ (i.e. on $\mathrm{m}_{\mathrm{z}}$ ), then, in formula (17), instead of parameter we may use its point evaluation $\delta_{z}^{\sim}$, which by the first receiver may be considered as permanent value. Then, confidence interval $(T)$ of vulnerability is defined by the confidence interval of $m_{z}$, which will be taken symmetrically from its point evaluation $m_{z}^{\sim}=m_{a}^{\sim}-m_{b}$, for the same probability $(\beta)$ for $m_{a}$ and $m_{b}$, the confidence interval can be defined by the same confidence probability $\left(I_{\beta}\right)$ for $m_{x}$. Thus, as the number of degrees of freedom ( $n-1)$ is reduced, the degree of indefiniteness to assess the mathematical expectation of random quantity $m$ increases, i.e. the confidence interval increases for the same confidence probability. Therefore, the number of degree of freedom for $m_{x}$ cannot be more than the minimum values of the numbers of degree of freedom for $m_{a}$ and $m_{b}$.

Thus, if taking $\beta$ as confidence probability and $K=\min \left(\mathrm{n}_{\mathrm{a}}\right.$, $\left.n_{b}\right)-1$ as a number of degrees of freedom for $m_{z}$, from the Student distribution equation, we can identify $t_{\beta}$, then from formula (16) we can identify $\varepsilon_{\beta}$ and consequently, the lower and upper limits of the confidence interval of $m_{1}$ and $m_{2}(16)$ :

$$
\varepsilon_{\beta}=t_{\beta} \sqrt{ } D^{\sim} /(K+1) ; m_{1}=m^{\sim}-\varepsilon_{\beta} ; m_{2}=m^{\sim}+\varepsilon_{\beta} .
$$

By inserting the values of $m_{1}$ and $m_{2}$ in formula (17), we will gain the confidence interval for the lower and upper limits for $T_{1}$ and $T_{2}$ vulnerability, with its confidence interval of $\beta$ :

$$
z_{1}=\Phi^{*}\left(-m_{1} / \sqrt{ } D^{\sim}{ }_{a}+D_{b}\right) ; \quad z_{2}=\Phi^{*}\left(-m_{2} / \sqrt{ } D_{a}^{\sim}+D_{b}^{\sim}\right)
$$

Thus, by $\beta$ probability, the value of vulnerability of the riverbank 
will not go beyond the interval:

$$
\mathrm{I}_{\beta}=\left[\Phi^{*}\left(-\left(\mathrm{m}^{\sim}-\varepsilon_{\beta}\right) / \sqrt{ } \mathrm{D}_{\mathrm{a}}^{\sim}+\mathrm{D}_{\mathrm{b}}^{\sim}\right), \Phi^{*}\left(-\left(\mathrm{m}^{\sim}+\varepsilon_{\beta}\right) / \sqrt{ } \mathrm{D}_{\mathrm{a}}^{\sim}{ }_{\mathrm{a}}+\mathrm{D}_{\mathrm{b}} \mathrm{b}\right)\right](21)
$$

\section{Results and Discussion}

With the aim of practical application of the considered method, let us examine a virtual example. Assume, we have the observation data subject to the normal distribution law. For the admissible river current velocity $\mathrm{n}_{\mathrm{a}}=15$, mathematical expectation $\mathrm{m}_{\mathrm{a}}=3 \mathrm{~m} \mathrm{sec}^{-1}$, mean square deviation $\sigma_{\mathrm{a}}=0.9$; for bed velocity, $n_{b}=20$. We must identify the confidence interval of the bank vulnerability.

To imitate the given random quantities, let us use the table of values of random quantities of Gauss, with its mathematical expectation of $a=0$ and its mean square deviation of $\sigma=1$.

It is easy to prove that random quantity ' $=a+\sigma$, which will subject to the normal distribution law, with mathematical expectation of $m\left[{ }^{\prime}\right]=\alpha$ and dispersion $D\left[{ }^{\prime}\right]=\sigma^{2}$.

By subsequent use of ' $=a+\sigma$, and Gauss table of values of random quantity, we will calculate the values of $\mathrm{V}_{\mathrm{a}}$ and $\mathrm{V}_{\mathrm{b}}$ :

$V_{a 1}=3+0,9 \cdot 0,2005 \approx 3.18$

$\mathrm{V}_{\mathrm{a} 2}=3+0,9 \cdot 1,1922 \approx 4.07 ; \ldots$

$\mathrm{V}_{\mathrm{b} 1}=4+1,3 \cdot 0,2005 \approx 4.26 ; \ldots$

$\begin{array}{llllllll}\mathrm{i} & 1 & 2 & 3 & 4 & 5 & 6 & 7 \\ \mathrm{Vdi} & 3,8 & 4,07 & 2,99 & 3,03 & 3,94 & 1,37 & 4,06 \\ 8 & 9 & 10 & 11 & 12 & 13 & 14 & 15 \\ 3,00 & 4,04 & 2,0 & 1,57 & 3,52 & 4,69 & 3,66 & 2,75 \\ \mathrm{i} & 1 & 2 & 3 & 4 & 5 & 6 & 7 \\ \mathrm{~V}_{4} & 4, & 26 & 5,55 & 3,93 & 4,04 & 5,35 & 1,64 \\ 8 & 9 & 10 & 11 & 12 & 13 & 14 & 15 \\ 5,50 & 4,00 & 5,53 & 3,13 & 1,93 & 4,76 & 6,45 & 4,96 \\ 16 & 17 & 18 & 19 & 20 & & & \\ 3,64 & 5,40 & 4,70 & 2,80 & 4,12 & 5,92 & & \end{array}$

According to the formulas (18) and (19) we have calculated the relevant values:

$\mathrm{m}_{\mathrm{a}}^{\sim}=48.27: 15=3.22 ; \quad \mathrm{D}_{\mathrm{a}}^{\sim}=12.2407: 14=0.874 ; \quad \delta_{\mathrm{a}}=0.935$

$\mathrm{m}_{\mathrm{b}}=87.78: 20=4.39 ; \mathrm{D}_{\mathrm{b}}=31.9652: 19=1.682 ; \delta_{\mathrm{b}}=1,297$

For random quantity $z=V_{a}-V_{b}$ we will have:

$m \sim z=m \sim a-m \tilde{b}=3.22-4.97=-1.17$
$D^{\sim} z=D^{\sim} a-D^{\sim} b=0.874+1.682=2.56$

$\delta_{z}=\sqrt{ } D_{z}^{\sim}=\sqrt{ } 2.56=1.6$.

From the formula (17) and tables of the values of distribution of normal function, we identify the z-point evaluation of the vulnerability of the river bank:

$\mathrm{z}=\Phi^{*}\left(-\left(\mathrm{m}_{\mathrm{z}} / \delta_{\mathrm{z}}\right)\right)=\Phi^{*}(-(1.17 / 1.6))=\Phi^{*}(0.73) \approx 0.77$.

Now, let us determine the confidence interval for $m_{z}$, with confidence probability of $\beta=0.95$. As $\min \left(n_{a}, n_{b}\right)=\min (15,20)=15$, then the degree of freedom will equal to $K=15-1=14$. From the table of distribution of the given Student fractiles $\beta$ and $K$ we find $t_{0.95}=2.14$. From here, by using the formula (19) we define:

$\varepsilon_{0.95}=t_{0.95} \sqrt{ } D_{z}^{\sim} /(K+1)=2.14 \sqrt{ } 2.56 / 15 \approx 0.88$

Consequently, the lower and upper limits of the confidence interval for values $m_{1}$ and $m_{2}$ will equal:

$m_{1}=m_{z}-\varepsilon_{0.95}=-1.17-0.88=-2.05$;

$m_{2}=m_{z}^{\tilde{z}}+\varepsilon_{0.95}=-1.17+0.88=-0.29$.

Consequently, from the table of the normal function distribution and formula (10) we identify $z_{1}$ and $z_{2}$ limits of the confidence interval for the vulnerability of a river bank:

$\mathrm{z}_{1}=\Phi^{*}\left(-\left(\mathrm{m}_{\mathrm{z}}{ }_{\mathrm{z}} / \delta_{\mathrm{z}}\right)\right)=\Phi^{*}(-(2.05 / 1.6))=\Phi^{*}(1.28)=0.9$

$\mathrm{z}_{2}=\Phi^{*}\left(-\left(\mathrm{m}_{\mathrm{z}}^{\sim} / \delta_{\mathrm{z}}{ }_{\mathrm{z}}\right)\right)=\Phi^{*}(-(0.29 / 1.6))=\Phi^{*}(0.18)=0.57$

Finally, the vulnerability of a river bank with 0,95 probability will be within the interval of $\mathrm{I}_{0.95}=(0.57 ; 0.9)$ with point evaluation $z=0.77$.

Among natural phenomena the freshet is such an event, which caused, causes and will cause major damage to population of any country; today there is no methodology, which allows us to forecast, with acceptable accuracy, the risks related to riverside degradation in order to timely carry out proper measures to avoid expected damage. Therefore, the mentioned phenomenon will remain the subject of research in an appropriate field of science for a long time.

The mechanism of riverside destruction by water flow is considered as a random process, which depends on both the effect of flow rate and riverside stability. Vulnerability, the characteristic of the riverside is taken as the indicator of this process with respect to such influence, and famous model "load stability" from the theory of reliability is used for its identification. Based on this fact, a result obtained via theoretical formalization in the form of represented formulas at this stage should be considered as rough approximation and cannot be considered as acceptable for attaining a high accuracy. 
Within the framework of initiating the research it was planned to continue working in order to improve existing deficiencies, in particular, determination of the limits of approximate values of vulnerability with preliminarily defined accuracy and taking the time factor into account that will be a huge step forward compared to the actual state of things characteristic of riverside.

\section{References}

Bazovsky, I.: Reliability Theory and Practice. Courier Corporation, Mathematics, p. 292 (2004).

Burrell, B.C., M. Huokuna, S. Beltaos, N. Kovachis, B. Turcotte and M. Jasek: Flood hazard and risk delineation of ice-related floods: Present status and outlook." In 18th CGUHS CRIPE Workshop on the Hydraulics of Ice Covered Rivers, Quebec City, Quebec Canada (2015)

De Philip, M. and T. Moberg: Ecosystem flow recommendations for the Susquehanna River basin. The Nature Conservancy, Harrisburg, Pennsylvania (2010).

Gertsbakh, I.: Reliability theory: With applications to preventive maintenance. Springer, p. 218 (2013).

Gnedenko, B.V., Y.K. Belyayev and A.D. Solovyev: Mathematical methods of reliability theory. Academic Press (2014).

Høyland, A. and M. Rausand: System reliability theory: models and statistical methods. Vol. 420. John Wiley \& Sons, p. 643 (2009).

Korkmaz, H., B. Çetin, V. Kuscu, I. Ege, A. Bom, E. Ozsahin and A. Karatas: Temporal changes of land use in Asi river delta (Hatay, Southern Turkey). J. Environ. Biol., 33, 463-473 (2012).

Jardine, A.K. and A.H. Tsang: Maintenance, replacement and reliability: Theory and applications. CRC Press, p. 339 (2013).
Lebel, L. and T.S. Bach: Risk reduction or redistribution? Flood management in the Mekong region. Asian J. Environ. Disaster Manag., 1, 1(2009).

Mirtskhulava, T.: The Dangers and Risks on Some Water and Other Systems. Tbilisi, Nauka, p. 53 (2003).

Oyer, Z.: Water infrastructure vulnerability to coastal flood hazards: A space-place analysis of Manteo, New Bern and Plymouth, North Carolina (2014).

Pielke, Jr. R.A.: Damaging floods as a framework for assessment. Floods, 1, 133 (2000)

Rashid, H.: Interpreting flood disasters and flood hazard perceptions from newspaper discourse: Tale of two floods in the Red River Valley, Manitoba, Canada. Applied Geography, 31, 35-45 (2011).

Rausand, M. and A. Høyland: System reliability theory: Models, Statistical Methods and Applications. John Wiley and Sons, p. 396 (2004).

Ronghui, L., W. Pan, J. Guo, Y. Pang, J. Wu, Y. Li, B. Pan, Y. Ji and L. Ding: Studies on kinetics of water quality factors to establish water transparency model in Neijiang River, China. J. Environ. Biol., 35, 513-519(2014).

Saint-Laurent, D.: Reconstruction of flood events and links with climatic factors: A case study of the Saint-François Basin. J. East. Townships Stud., 31, 5(2007).

Simeoni, U. and C. Corinne: A review of the Delta Po evolution (Italy) related to climatic changes and human impacts. Geomorphology, 107,64-71 (2009).

Shannon, S. B.: Natural and unnatural complexities: Flood control along Manitoba's Assiniboine River. J. Histor. Geogr., 36, 57-67 (2010).

Thoft-Cristensen, P. and M.J. Baker: Structural reliability theory and its applications. Springer Science and Business Media, p. 266 (2012) 